\title{
A New Classification of Spreader Flap Techniques
}

\author{
Jochen Wurm, MD ${ }^{1}$ Milos Kovacevic, $\mathrm{MD}^{2}$ \\ ${ }^{1}$ Department of Otolaryngology, Head and Neck Surgery, University \\ Erlangen-Nuremberg, Erlangen, Germany \\ 2 Praxis am Hanse-Viertel, Hamburg, Germany \\ Address for correspondence Jochen Wurm, MD, Department of \\ Otolaryngology, Head and Neck Surgery, University Erlangen- \\ Nuremberg, Waldstr. 1, Erlangen 91054, Germany \\ (e-mail: jochen.wurm@uk-erlangen.de).
}

Facial Plast Surg 2013;29:506-514.

\begin{abstract}
Keywords

- spreader flaps

- internal nasal valve

- middle vault

- inverted $v$ deformity

The objective of this study was to introduce various spreader flap technique modifications to adjust the width of the middle nasal vault in patients who underwent rhinoplasties with humpectomy. Decisive modifications of current spreader flap techniques were performed to allow a more natural restoration of the middle nasal vault and the internal nasal valve after humpectomy. Additional steps provide tools to adjust the width and shape of the middle nasal vault according to patients' requirements. The techniques were categorized into "basic spreader flaps," "flaring spreader flaps," "support spreader flaps," and "interrupted spreader flaps." The various spreader flap techniques were used during 576 primary septorhinoplasties in patients with hump noses, hump/crooked noses, or hump/tension noses. The average follow-up was 19 months. Patients who received basic spreader flaps or a flaring spreader flaps tended to show a slightly too wide middle nasal vault, revision surgery was necessary in four of these cases. All other patients showed an appropriate width in the middle nasal vault and an aesthetically pleasing course of the dorsal aesthetic lines. No signs of inverted $v$ deformities or collapse of the internal nasal valve were observed in any of the patients. Patients who had reported impaired nasal breathing preoperatively described clearly improved subjective symptoms. The described techniques appear to be appropriate and highly promising as a supplement to existing procedures for reconstructing the middle nasal vault and internal nasal valves. No additional cartilage grafts are needed.
\end{abstract}

Removal of a nasal hump and reduction of the dorsum during rhinoplasty loosens or resects the anatomic connections between the upper lateral cartilages and the septum and bone. The structural arrangement of the middle nasal vault is thus lost. If reconstructive measures are not performed, this often leads to adverse functional and aesthetic sequelae. The lateral walls of the nose and the internal nasal valves may collapse, leading to nasal obstruction. A typical external sign of a collapsed middle nasal vault is the formation of the "inverted v" deformity, with destruction of the natural contours and interruption of the aesthetic lines of the nasal dorsum (- Fig. 1) ${ }^{1}$

In patients with a high and narrow nose, a finding of a collapsing lateral nasal wall and collapse of the internal nasal valves is often easily recognizable preoperatively. By contrast, it is more difficult to assess cases in which there are initially no obvious pathological findings in the middle nasal vault but in which there is a high risk of collapse of the internal nasal valves and development of an inverted v deformity. Patients with short nasal bones, thin skin, and weak upper lateral cartilages fall into this category. The aim in these cases is to maintain or restore normal functional and aesthetic anatomy through rhinoplasty.

Implantation of spreader grafts is regarded as the standard method for preventing collapse of the internal nasal valves and an inverted $v$ deformity. In functional terms, the grafts move the upper lateral cartilages away from the septum and enlarge the internal nasal valve. In addition, the volume and
Issue Theme Nasal Septal Reconstruction; Guest Editor, Fazil Apaydin, MD
Copyright (c) 2013 by Thieme Medical Publishers, Inc., 333 Seventh Avenue, New York, NY 10001, USA. Tel: +1(212) 584-4662.
DOI http://dx.doi.org/ 10.1055/s-0033-1360596. ISSN 0736-6825. 


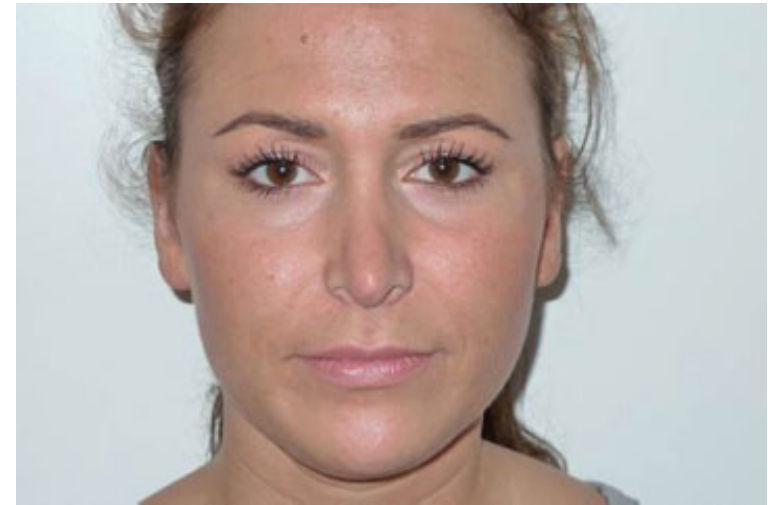

Fig. 1 Typical appearance of an inverted $v$ deformity with collapse of the middle vault.

supportive function of spreader grafts give the middle nasal vault harmonious and natural contours. ${ }^{1-6}$

The large number of different techniques that are used underlines the importance of maintaining or restoring this region. In particular, "spreader flaps" and their modifications have been attracting increasing attention in the more recent literature on the topic. First, the technique was described by Oneal and Berkowitz in 1998..$^{7}$ The authors considered spreader flaps as an alternative to preserve the excess height of the upper lateral cartilages and convert it to horizontal flaps to restore the normal t shape of the cartilaginous vault. In principle, the upper lateral cartilages are not resected during humpectomy, but separated from the septum, mobilized, and then rotated into the area of the internal nasal valves. By positioning the excessive cartilages along both sides of the cranial septum, the flaps act as spreader grafts by preserving or restoring the middle nasal vault. With spreader flaps, there is no need to harvest additional graft material from the septum.

Various authors have described the use of spreader flaps as a substitute for traditional spreader grafts in patients with nasal humps during primary rhinoplasty. ${ }^{7-12}$ However, traditional spreader flap techniques only allow restoration of the natural shape of the middle nasal vault to a limited extent. More important, present spreader flap techniques offer only a limited alteration or adjustment of the width of the middle nasal vault.

We have developed additional modifications of the spreader flap technique that offer a more natural reconstruction of the middle nasal vault. Providing a series of different options to alter the width of the middle nasal vault, we suggest a new classification of spreader flap techniques to either widen or narrow this important region.

\section{Methods}

\section{Basic Spreader Flap}

This method can be used both with an endonasal or an external (open) approach. The present authors prefer the external approach, and therefore, all further details given here refer to this procedure.
Initially, separation of the mucosal layers from the septum and adjoining upper lateral cartilages is necessary. This can be performed via a hemitransfixion incision during the septal correction. Starting from the anterior septal angle, additional mucosal tunnels are established below the upper lateral cartilages and below the bony nasal skeleton. After opening of the nose and separation of the nasal dorsum, detachment of the lateral cartilages from the septum before humpectomy represents a decisive step. The perichondrium of the upper lateral cartilages along a strip 0.5 to $1.0 \mathrm{~cm}$ wide over their whole length and adhesions of the lateral cartilages to the nasal bones at the roof of the nose are locally released (-Fig. 2 ).

The intended reduction of the nasal dorsum is performed now. This allows complete preservation of the upper lateral cartilages. With the mobilization method described, the lateral cartilages can be rotated medially and positioned bilaterally alongside the septum in the area of the internal nasal valve.

Up to this stage, the described surgical steps comply with previously published spreader flap techniques and describe nothing new. But our method in securing the spreader flaps to the septum marks a crucial difference. If at all, previous techniques use holding sutures that run through the apex of the spreader flaps which in our opinion leads to an
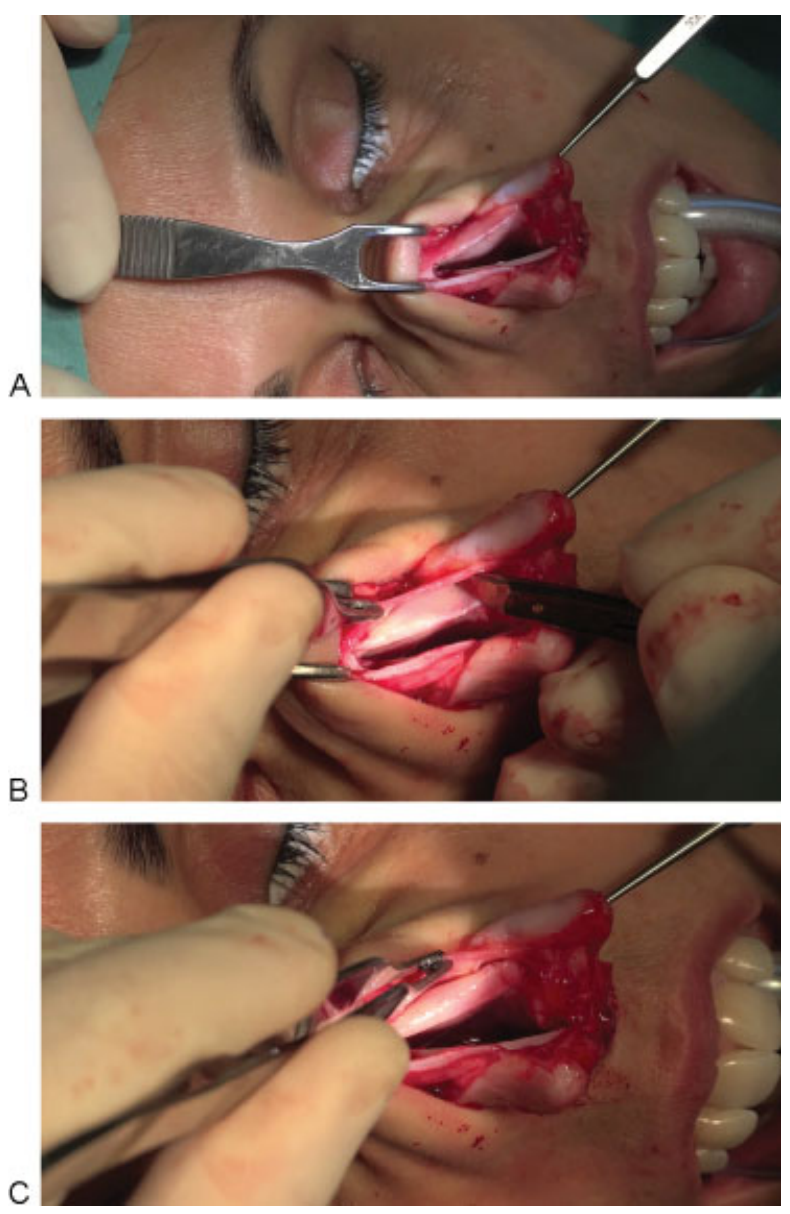

Fig. 2 (A) Upper lateral cartilages separated from the septum. (B) Releasing the perichondrium from the upper lateral cartilage. (C) Exposed upper lateral cartilage. 
A
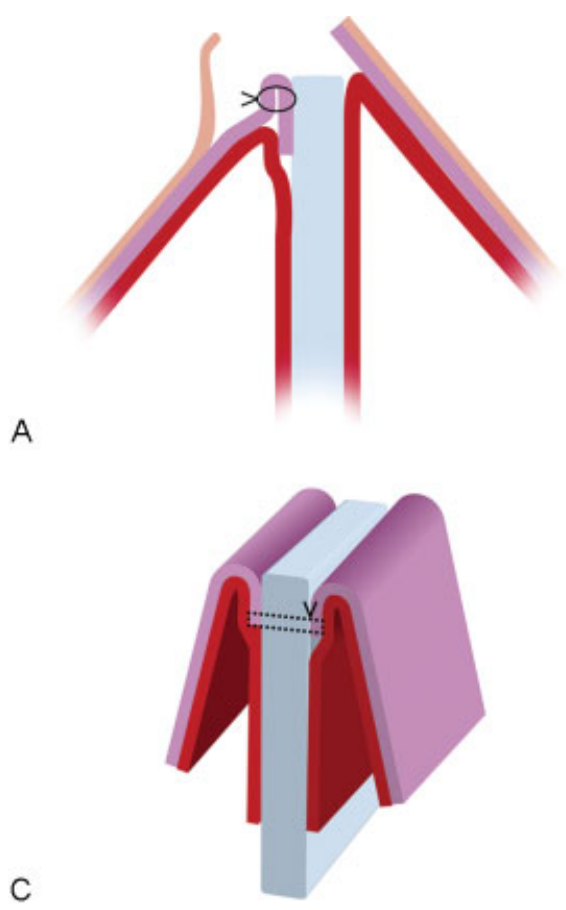
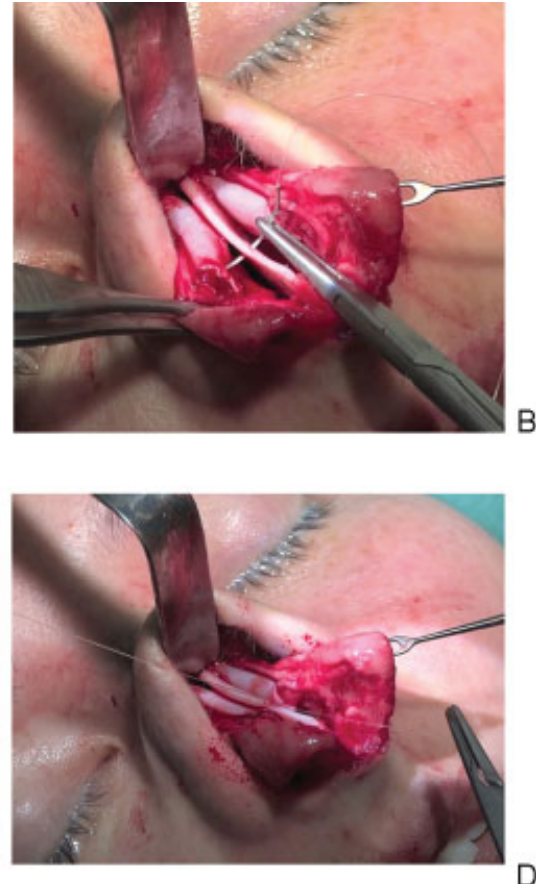

Fig. 3 (A) Conventional spreader flap suture technique. (B) "Anchoring suture" in situ. (C) Diagram of "anchoring suture" to fix the upper lateral cartilage to the septum. (D) Bilateral basic spreader flaps in situ.

unnecessary squeezing of the flaps and narrowing of the internal nasal valve (-Fig. $\mathbf{3 A})^{8-10,13}$

In contrast to this, we place our suture only through the rotated cartilage parts and then the cranial septum subsequently. This approach not only avoids any squeezing and narrowing but also preserves the natural tension and shape of the cartilages (-Fig. 3B). This "anchoring suture" is usually not made separately for each side, but in the form of a mattress suture that grasps both upper lateral cartilages and the cranial septum (-Fig. 3C, D). In principle, several "anchoring sutures" are possible, if needed, to fix basic spreader flaps in place. Usually, however, one suture of this type at the distal end of the upper lateral cartilages provides sufficient stability. The combination of rotated spreader flaps and the "anchoring sutures" is called "basic spreader flap." - Fig. 3C, D shows the placement of bilateral basic spreader flaps; in principle, the same technique can also be used unilaterally.

To begin with the basic spreader flap, the procedure shown thus provides as natural as possible a reconstruction of the middle nasal vault and internal nasal valves. Furthermore, the basic spreader flaps create a kind of starting situation that allows the assessment if either additional narrowing or widening is required.

Depending on the individual findings and the shape and quality of the upper lateral cartilages, the supplementary methods described below then provide additional options to adjust the width of the middle nasal vault.

\section{Flaring Spreader Flap}

Flaring spreader flaps can be used in cases in which widening of the middle nasal vault is required after the creation of basic spreader flaps. If the cartilage is sufficiently stable, this modification can also be used as a support for the nasal bones when osteotomies have been performed.

A horizontal mattress suture grasps both upper lateral cartilages and the upper edge of the septum cranially and caudally (-Fig. 4). Depending on how much traction is applied to the mattress suture, the lateral cartilages open to a greater or lesser extent, like a pair of wings. This makes it possible to adjust the enlargement of the middle nasal vault to the desired degree.

Depending on the findings in the individual patient, this suture technique can be used for individual optional variations. The location of the suture and the width of the cartilage surface grasped allow enlargement of the middle nasal vault at various points, on the one hand. On the other hand, placing several sutures also allows additional fine adjustment. A flaring spreader flap can also be used unilaterally and in combination with other spreader flap techniques.

\section{Support Spreader Flap}

In many cases, the upper lateral cartilages are relatively wide after detachment from the septum, or show marked asymmetry or excessive bulging due to the underlying pathology. After placement of the basic spreader flaps using the anchoring suture, a nasal dorsum that is aesthetically too wide, or asymmetric nasal dorsum lines, may therefore arise. In these patients, modeling of the lateral cartilage in the affected region is indicated.

For this purpose, basic spreader flaps are initially prepared with the anchoring suture, as described above. In the region that still has asymmetries or protrusions, a mattress suture is then tied through the lateral cartilage and the cranial edge of the septum. This can be done either unilaterally or bilaterally. With the appropriate traction, persisting protrusions or asymmetries 
A

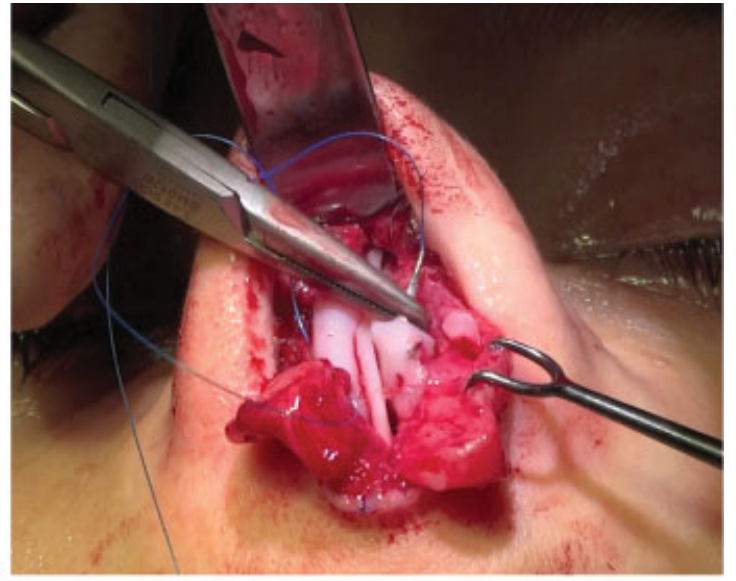

B

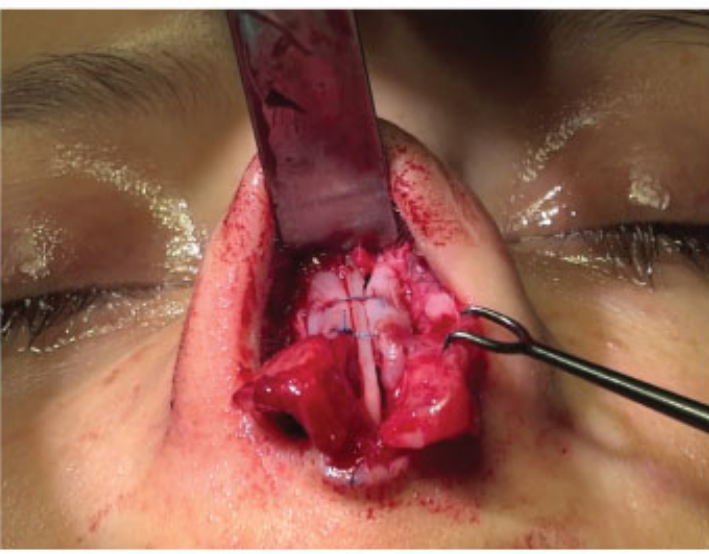

C

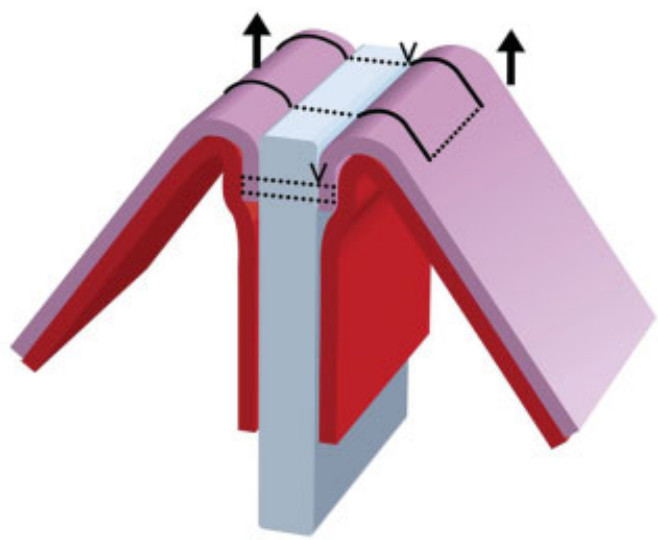

Fig. 4 (A) Horizontal mattress suture to create flaring spreader flaps. (B) Flaring spreader flaps in situ. (C) Diagram of bilateral flaring spreader flaps.

can be corrected and the width of the middle nasal vault can be regulated. If the dorsum is particularly wide, carrying out these dissections mainly in the caudal parts of the upper lateral cartilage can be recommended to achieve the aesthetically favorable canoe shape. A combination of the basic spreader flap and an additional modeling mattress suture is called a “support spreader flap" (-Fig. 5A, B).

This technique can also be used in patients with a moderately crooked nose and only a unilateral impression on the nasal dorsum. In these patients, in whom the contralateral

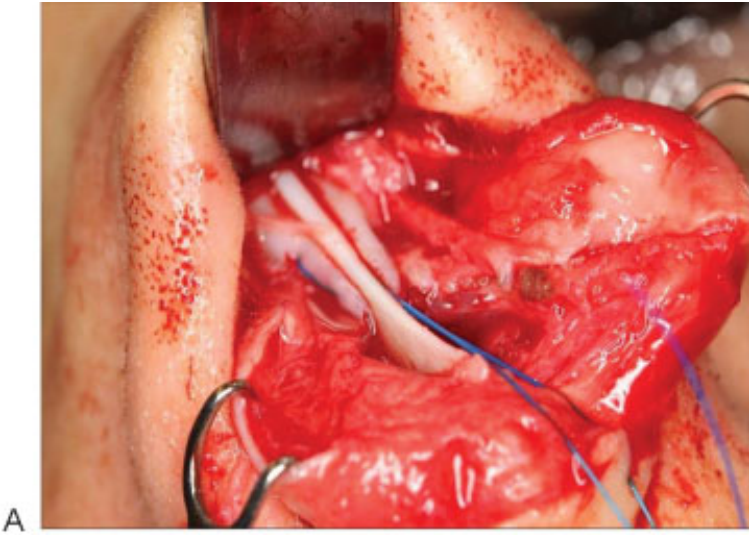

B
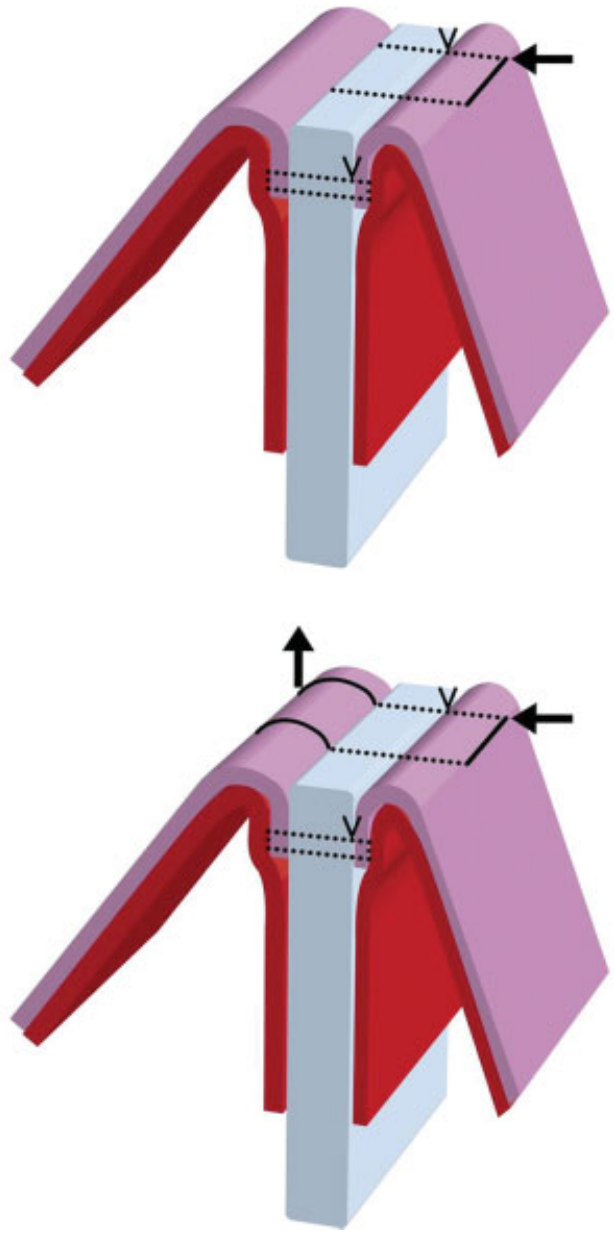

Fig. 5 (A) Bilateral support spreader flap in situ. (B) Diagram of unilateral support spreader flap. (C) Combination of support and flaring spreader flap.

side of the nose runs almost parallel to the aesthetic nasal dorsum lines, osteotomies are only performed on the side with the impression. The nasal bone with the unilateral osteotomy is then lateralized, and a flaring spreader flap is created. The flaring spreader flap serves here on the one hand as a placeholder to avoid retrusion of the bone, and on the other hand, it enables the internal nasal valve to be enlarged, improving the air supply. Contralaterally, a support spreader flap is placed to adapt the shape and width of the nasal dorsum accordingly (-Fig. 5C). 


\section{Interrupted Spreader Flap}

In a fairly small number of patients, excessively large and thick upper lateral cartilages or areas with fracture lines, kinking, and scarring are present. In these cases, it is often not possible, even with the support spreader flap method, to achieve an aesthetically pleasing width in the nasal dorsum and symmetrical nasal dorsal lines. However, single or multiple stab incisions into these cartilage areas make it possible to weaken the rigidity of the cartilage at points ( $\mathbf{F i g . 6 A , ~ B ) . ~}$ The lateral cartilage is not completely transected by the selective stab incisions. After selective incisions, it is easier to give the locally weakened lateral cartilage the desired shape with the subsequent mattress suture. However, sometimes bulging of the upper lateral cartilages reaches underneath the nasal bones. After osteotomies, these cartilage portions tend to push the nasal bones outward creating a deviated nose or a too wide dorsum. In these cases, we place the incision at the cranial end of the upper lateral cartilage to reduce its force to the nasal bones. A second incision is made at the caudal end of the upper lateral cartilage to create a favorable canoe shape of the dorsum (-Fig. 6C). The combination of basic spreader flap, selective stab incisions, and a modeling mattress suture is called the "interrupted spreader flap."

The various spreader flap techniques were used during primary septorhinoplasties between 2009 and 2012. In a retrospective study, the results of 374 patients with hump noses, 115 with hump/crooked noses, and 87 with hump/ tension noses were reviewed by both authors. Thirty-four procedures involved basic spreader flaps with no additional measures, 164 were flaring spreader flaps (72 of which were unilateral), and 361 procedures involved support spreader flaps (43 of which were unilateral). Seventeen patients received interrupted spreader flaps. The patients consisted of 403 women and 173 men, aged 17.5 to 63 years. The followup range was between 5 and 26 months with an average follow-up of 19 months.

Particular attention was given to the shape of the middle nasal vault and the dorsal aesthetic lines. No rhinomanometry studies were performed.

\section{Results}

At the beginning of the study, the authors mainly used the basic and flaring spreader flap methods. During the course of the study, however, it was found that patients with only hump noses or with hump/tension noses tended to have a slightly too wide middle nasal vault. The enlargement was much greater, with unsatisfactory cosmetic results, in three cases after flaring spreader flaps and in one case after creation of a basic spreader flap. Revision procedures were necessary in these patients after 1 year. Successful correction of the excessive width of the middle nasal vault was possible by converting the spreader flaps previously used into support spreader flaps.

Following these experiences, a much larger number of support spreader flaps were therefore used during the subsequent period. Patients treated with this technique had an
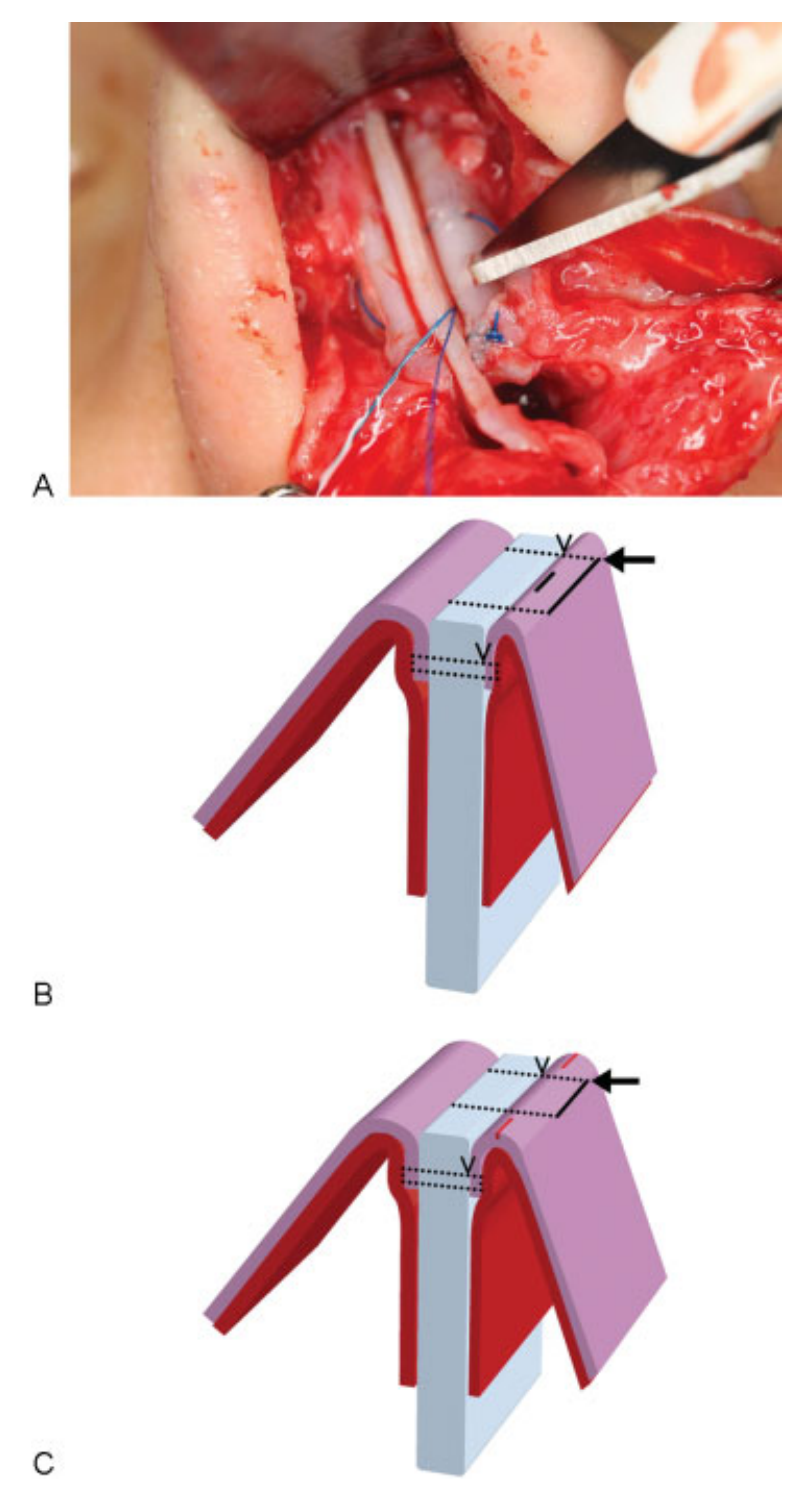

Fig. 6 (A) Punctual incision of upper lateral cartilage to create an interrupted spreader flap. (B) Diagram of unilateral interrupted spreader flap. (C) Punctual incision of upper lateral cartilage at cranial and caudal end.

appropriate width in the middle nasal vault and an aesthetically pleasing course of the nasal dorsal lines. Slight asymmetries in the width of the cartilaginous nasal slopes occurred in four patients, but revision procedures were not required. In one patient, there was a slight depression in the dorsum in the area of the junction between the bony and cartilaginous nasal dorsum. In this case as well, no further measures were performed at the patient's request.

Among those patients who received support spreader flaps, we observed a recurrent deviation of the nasal bones to one side in six cases. Revision surgery was performed in all cases. Intraoperatively, we found a bulging of the cranial parts of the upper lateral cartilages that reached underneath the nasal bones. After osteotomies, these cartilage parts obviously pushed the mobile nasal bones outward creating the recurrent deviation of the nose. Thus, the modeling mattress 
suture alone was considered not powerful enough to prevent the movement of the bones. The problem was solved by an additional vertical incision placed at the cranial and caudal end of the upper lateral cartilages ( $\mathbf{- F i g}$. 6C) and reosteotomies.

Taking all spreader flap techniques described into account, no signs of inverted $v$ deformities or collapse of the internal nasal valve were observed in any of the patients. Patients who had reported impaired nasal breathing preoperatively described improved subjective symptoms.

The modification of flaring spreader flaps for patients with moderate crooked noses and unilateral impression of the nasal dorsum was used in nine cases. The combination of only unilateral osteotomies with placement of a flaring spreader flap on the side with the impression and placement of a support spreader flap on the contralateral side made it possible to shape and maintain a straight nasal dorsum in all cases.

The use of flaring spreader flaps or modifications of them did not appear adequate intraoperatively in three patients during the correction of more pronounced crooked noses. In these cases, a unilateral spreader graft was implanted additionally to splint the concave side of the nose.

The overall revision rate was $7.8 \%$. Revision surgery was necessary due to occurring tip asymmetries, irregularities at the dorsum, or re-deviation of the bony vault in severely crooked noses. Concerning the spreader flap techniques and the cartilaginous middle vault revisions had to be performed only in those cases already mentioned above.
No complications involving infection, excessive swelling, or hemorrhage occurred with the method. Examples of representative results following the use of the various spreader flap techniques are shown in -Figs. 7-9.

\section{Discussion}

The middle vault of the nose is defined as the section between the caudal ends of the bony nasal skeleton and the cranial margins of the lower lateral cartilages. Its stability is determined by the T-shaped configuration formed by the upper lateral cartilages and the cartilaginous part of the septum. Nasal humps usually have both bony and cartilaginous elements. The extent of the bony components and thus the length of the cartilaginous middle nasal vault vary depending on the length of the nasal bones.

Traditional en bloc resection of a prominent nasal hump weakens the stability of the middle nasal vault. In addition to causing poorer nasal breathing due to collapse of the internal nasal valves, typical adverse sequelae also include development of an inverted $v$ deformity, asymmetries on the lateral nasal walls, and interruption of aesthetic nasal dorsum lines. ${ }^{11,14}$

Implantation of spreader grafts has been regarded as the standard method of reconstructing the middle nasal vault since the technique was first described by Sheen. ${ }^{1}$ However, the use of spreader grafts involves some potential risks. Harvesting of the grafts can potentially weaken the stability
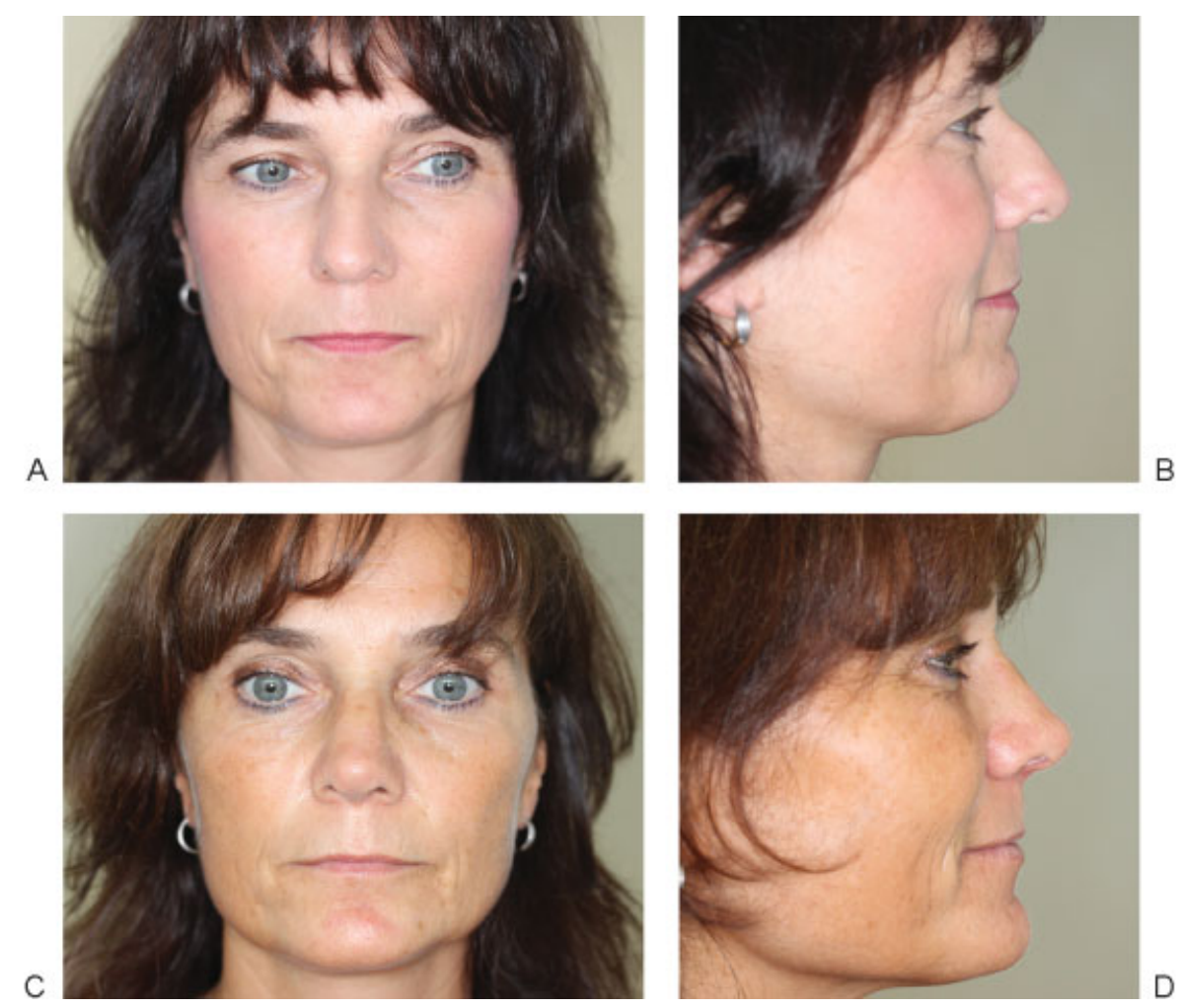

Fig. 7 (A,B) Patient with nasal hump and deviated nose to the right side. (C,D) Results 14 months postoperatively after bilateral flaring spreader flaps. 
A
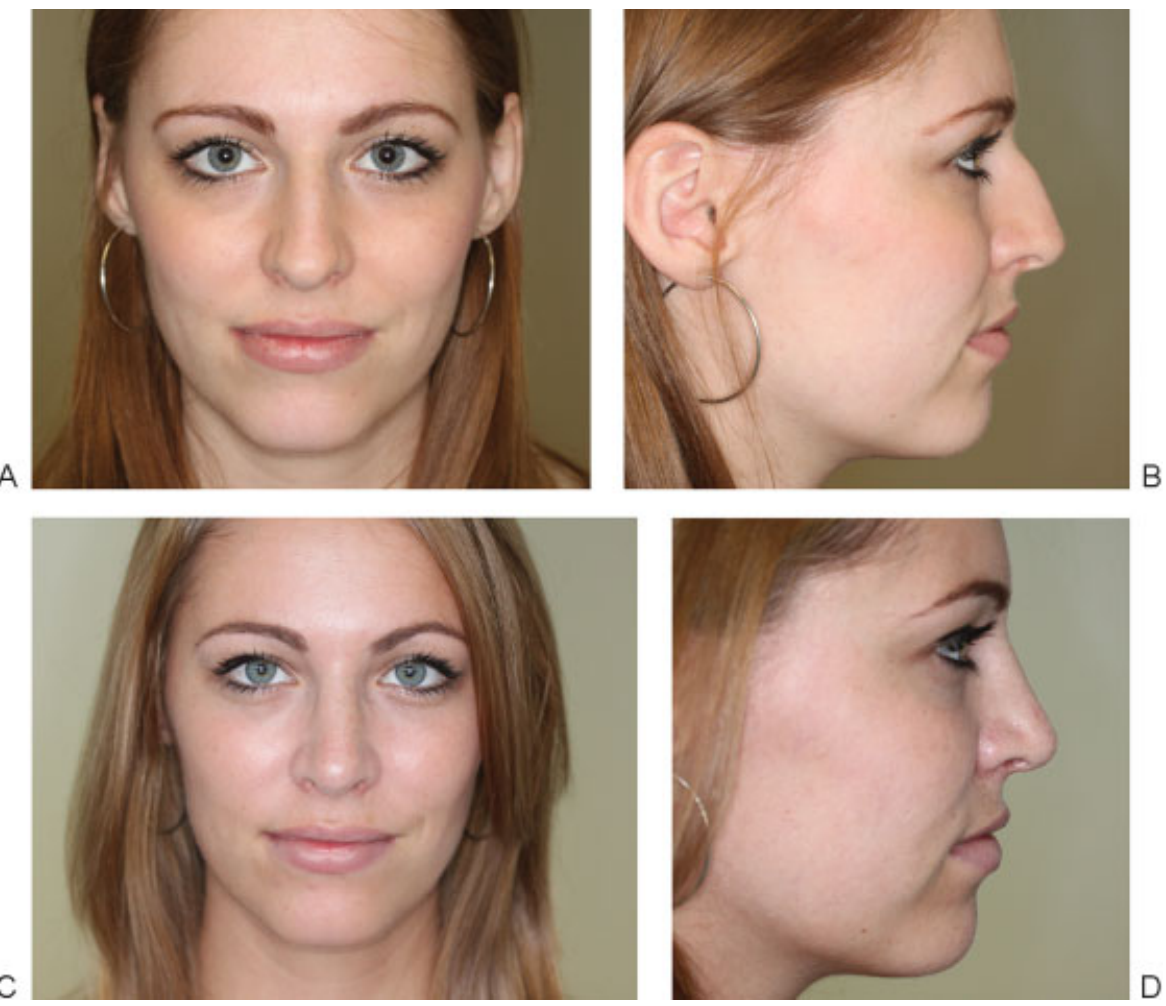

Fig. 8 (A,B) Patient with nasal hump and deviated nose to the left side. (C,D) Results 16 months postoperatively after bilateral support spreader flaps.

of the septum, and implanted spreader grafts may shift and become visible on the nasal dorsum., 8,15

Techniques have been developed to maintain the natural anatomy and function of the upper lateral cartilages without having to depend on additional harvesting of cartilage grafts. Oneal and Berkowitz described the spreader flap method and suggested that obvious indications for this technique would be cases in which excessive vertical length of the upper lateral cartilages in patients with hump noses can be reshaped into the desired horizontal width in the middle nasal vault. ${ }^{7}$

The method was taken up and modified by other authors. Byrd et al performed incisions along the longitudinal axis of the lateral cartilage, with preservation of the mucoperichondrium. The detached cartilage parts were turned inward as
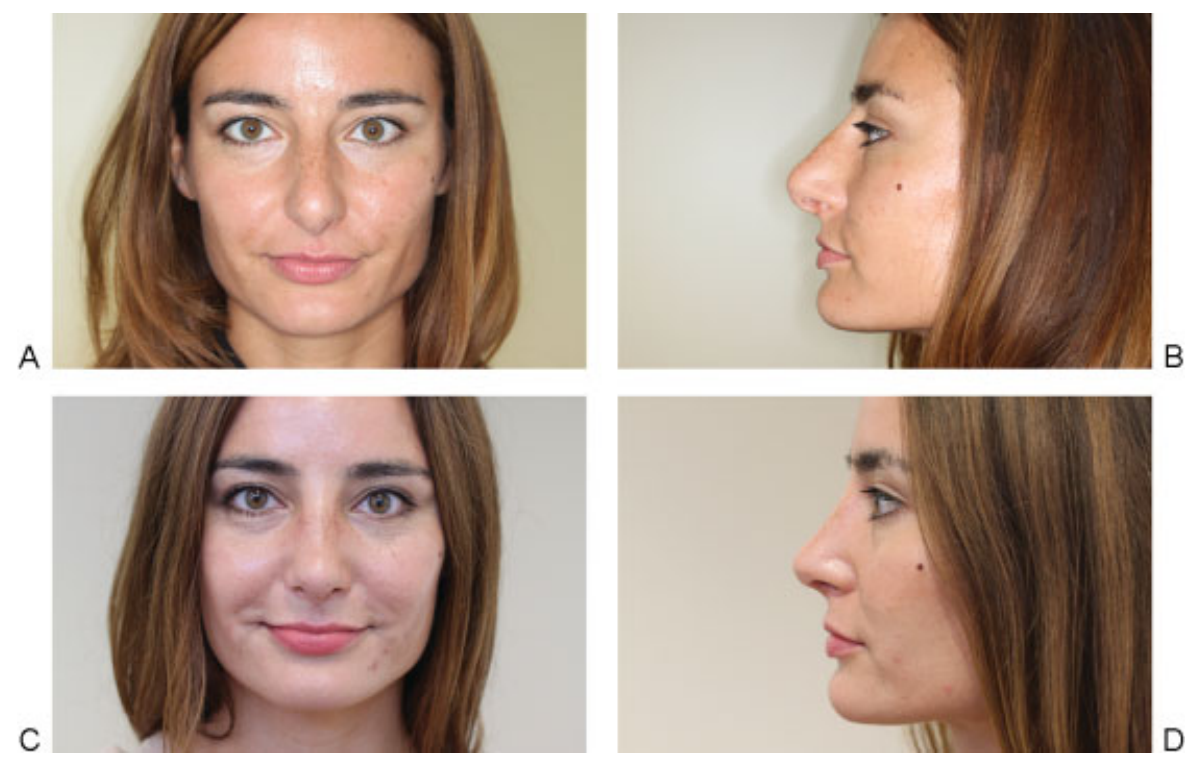

Fig. 9 (A,B) Patient with nasal hump and impression of the nasal side wall on the left. (C,D) Results 11 months postoperatively after flaring spreader flap on the right side and support spreader flap on the left side. 
spreader flaps between the septum and the remaining upper lateral cartilages. The attached mucoperichondrium served for stabilization, without additional fixing sutures. ${ }^{13}$

Gruber et al initially performed scoring of the cartilage to make it easier to turn in the spreader flaps. The authors later reduced the scoring of the cartilage to a minimum to prevent postoperative development of spreader flaps that were too narrow. ${ }^{9,10}$

In the view of the present authors as well, scoring and incisions, and particularly detachment of the lateral cartilage along its whole length, inevitably lead to weakening of the cartilage. In addition, the lateral cartilages lose the tension created by the turning of the cartilage. The risk of collapse of the internal nasal valve is therefore increased again.

In contrast to Byrd et al, most authors fix the rotated spreader flaps in place using sutures to the cranial parts of the septum. However, in all of the published reports, these sutures are led through the apex of the spreader flapsprecisely the region in which the natural vault is meant to be restored. In our view, this method of suturing leads to an unnecessary squeezing of the cartilages and therefore weakens the effect created by the spreader flaps again. We regard this as representing a crucial difference from the method presented here. When the sutures are positioned only between the rotated spreader flaps and the cranial septum (-Fig. 3), the cartilage again forms a natural vault and enlarges the region of the internal nasal valves. This suturing technique is certainly technically more demanding, but it appears to be successful in preventing inverted $v$ deformity.

The combination of the rotated spreader flap and the anchoring suture is called the "basic spreader flap" and serves as a stabilizing framework for reconstructing the middle nasal vault. In contrast to previously described spreader flap techniques, the modifications presented here now allow fine adjustments to be made in this region. When the basic spreader flap is used as a starting point, it is possible for the first time to control the width of the middle nasal vault depending on requirements, without the need for additional grafts.

"Flaring spreader grafts" can be used as a method of enlarging the middle nasal vault in patients with a very narrow nasal dorsum. This is evidently a very powerful technique, since in our experience there was a tendency to form a slightly too wide nasal dorsum, so that revision procedures were needed in four patients. This modification can therefore be recommended particularly in patients with a very high, narrow nasal dorsum and thin skin.

Park described the placement of what is known as a "flaring suture" as a supportive vertical mattress suture in addition to the implantation of spreader grafts for reconstructing the internal nasal valves. ${ }^{16}$ In contrast to this, we use horizontal mattress sutures. In our view, this allows the required cartilage surface to be grasped and expanded more variably.

"Support spreader flaps" and "interrupted spreader flaps," by contrast, are suitable for narrowing the middle nasal vault after creation of the "basic spreader flap." Depending on the rigidity and configuration of the cartilage, narrowing can be achieved here with sutures alone ("support spreader flaps") or in combination with selective cartilage incisions ("interrupted spreader flaps"). In contrast to the suturing method described by Gruber et al, however, the mattress sutures in support spreader flaps are not used to fix the spreader flaps in place but rather for fine adjustment, so that they weaken the structure of the middle nasal vault only insignificantly, if at all. ${ }^{9,10}$ The selective cartilage incisions used with the interrupted spreader flaps are also decisively different from the techniques described by Byrd et al and Gruber et al. In interrupted spreader flaps, the cartilage incisions are not performed along the whole length of the lateral cartilage, but only at points where the cartilage shows marked bulging or excessive thickness. ${ }^{9,10,13}$ This avoids weakening of the internal nasal valves previously reconstructed with the basic spreader flap. In our experience, this aggressive procedure also only appears to be indicated in a small number of patients.

In some patients with moderate crooked noses, preoperative analysis only shows unilateral impression of the nasal dorsum. If the contralateral nasal dorsum runs almost parallel to aesthetic dorsal lines, then a combination of flaring spreader flaps and support spreader flaps is an appropriate option for straightening of the nose-avoiding additional osteotomies on the contralateral side. To ensure that the nasal dorsum on the nonosteotomy side is not too wide after creation of the basic spreader flap, the support spreader flap technique can be used to adjust the width of the nasal dorsum there to correspond to that on the osteotomy side.

In patients with a marked crooked nose, residual deviation of the cartilaginous nasal dorsum may persist even after correction of the septum and appropriate osteotomies in the bony nasal skeleton. In these cases, the spreader flap techniques described here do not appear to be capable alone of compensating for the existing slant. In these conditions, greater thickness and stability in the cartilage are obviously needed to splint the nasal dorsum. In these patients, it is, therefore, necessary to resort to the traditional implantation of spreader grafts.

Typical indications for the use of the spreader flap techniques described here are seen in patients with hump noses, hump/tension noses, and slight to moderate hump/crooked noses. The methods can be used in patients with these diagnoses in whom septal corrections have already been performed and insufficient cartilage material is available in the septum. No additional grafts are needed for reconstruction of the middle nasal vault or the internal nasal valves.

We would propose a new classification of the various spreader flap techniques (-Fig. 10). Basic spreader flaps

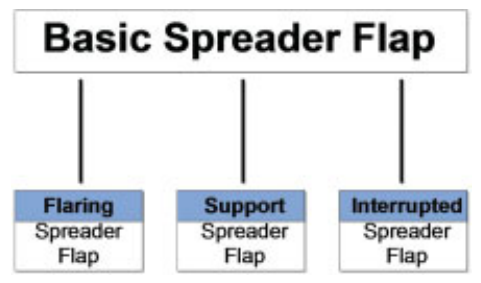

Fig. 10 Diagram of suggested new classification in spreader flap techniques. 
primarily serve to restore and stabilize the middle nasal vault and thus represent the basis for possible additional measures that can be performed. Flaring spreader flaps, support spreader flaps, and interrupted spreader flaps provide the surgeon with tools for fine adjustment of the middle nasal vault in accordance with the patient's individual requirements. An additional advantage is that the various techniques can be used either unilaterally or bilaterally, and also in combination with each other.

As the spreader flap techniques described here are new, with a maximum follow-up period of 26 months, long-term results are, of course, not yet available. However, the typical adverse sequelae of inverted $v$ deformity and collapse of the middle nasal vault following humpectomy have not yet been observed in any of the patients to date. The methods, therefore, appear to be appropriate and highly promising as a supplement to existing procedures for reconstructing the middle nasal vault and internal nasal valves.

\section{References}

1 Sheen JH. Spreader graft: a method of reconstructing the roof of the middle nasal vault following rhinoplasty. Plast Reconstr Surg 1984;73(2):230-239

2 Fischer H, Gubisch W. Nasal valves-importance and surgical procedures. Facial Plast Surg 2006;22(4):266-280

3 Ingels KJ, Orhan KS, van Heerbeek N. The effect of spreader grafts on nasal dorsal width in patients with nasal valve insufficiency. Arch Facial Plast Surg 2008;10(5):354-356
4 Riedel F, Bran G. [Cartilage grafts in functional and aesthetic rhinoplasty]. HNO 2008;56(2):185-198, quiz 199-200

5 Rohrich RJ, Hollier LH. Use of spreader grafts in the external approach to rhinoplasty. Clin Plast Surg 1996;23(2):255-262

6 Sykes JM. Management of the middle nasal third in revision rhinoplasty. Facial Plast Surg 2008;24(3):339-347

7 Oneal RM, Berkowitz RL. Upper lateral cartilage spreader flaps in rhinoplasty. Aesthet Surg J 1998;18(5):370-371

8 Neu BR. Use of the upper lateral cartilage sagittal rotation flap in nasal dorsum reduction and augmentation. Plast Reconstr Surg 2009;123(3):1079-1087

9 Gruber RP, Park E, Newman J, Berkowitz L, Oneal R. The spreader flap in primary rhinoplasty. Plast Reconstr Surg 2007;119(6): 1903-1910

10 Gruber RP, Perkins SW. Humpectomy and spreader flaps. Clin Plast Surg 2010;37(2):285-291

11 Ozmen S, Ayhan S, Findikcioglu K, Kandal S, Atabay K. Upper lateral cartilage fold-in flap: a combined spreader and/or splay graft effect without cartilage grafts. Ann Plast Surg 2008;61(5): 527-532

12 Rohrich RJ, Muzaffar AR, Janis JE. Component dorsal hump reduction: the importance of maintaining dorsal aesthetic lines in rhinoplasty. Plast Reconstr Surg 2004;114(5):1298-1308, discussion 1309-1312

13 Byrd HS, Meade RA, Gonyon DL Jr. Using the autospreader flap in primary rhinoplasty. Plast Reconstr Surg 2007;119(6):1897-1902

14 Guyuron B, Michelow BJ, Englebardt C. Upper lateral splay graft. Plast Reconstr Surg 1998;102(6):2169-2177

15 Aziz ZS, Brenner MJ, Putman HC III. Oblique septal crossbar graft for anterior septal angle reconstruction. Arch Facial Plast Surg 2010;12(6):422-426

16 Park SS. The flaring suture to augment the repair of the dysfunctional nasal valve. Plast Reconstr Surg 1998;101(4):1120-1122 